At the end of the review, $48 \%$ of the treatments were still in use, while $52 \%$ had stopped for various reasons: $20 \%$ ineffectiveness, $12 \%$ side effects, $12 \%$ maintained complete remission. The remaining low percentage was due to death, pregnancy, or study entry.

The side effects that led to a change in treatment was: non-immune hematological disorders in $4.1 \%$ (14\% of tocilizumab, $7 \%$ of rituximab and $6 \%$ of vedolizumab), immune disorders in $3.8 \%$ (6\% of secukinumab, $5 \%$ of tocilizumab and $4.5 \%$ of certolizumab), nervous system involvement in $2.4 \%$ ( $11.5 \%$ of golimumab, $10 \%$ of abatacept, $5 \%$ of tocilizumab), debut or worsening of heart failure in $1.4 \%(9,5 \%$ of tocilizumab, $2.5 \%$ of etanercept and $1.4 \%$ of adalimumab) and cancer-related in $0.7 \%(2 \%$ of infliximab, $1.7 \%$ of etanercept)

Conclusion: In these 10 years of follow-up and and evaluating 847 treatments, there were $10 \%$ hospitalizations due to infections $(n=86)$ causing death in 5 patients. In light of these results, and pending an exhaustive statistical analysis, we did not find high frequencies of serious side effects in our series. Very little long-term evidence exists on the safety of these drugs.

REFERENCES:

[1] Singh JA, Wells GA, Christensen R, et al. Adverse effects of biologics: a network meta-analysis and Cochrane overview. Cochrane Database Syst Rev. 2011;2011(2):CD008794. Published 2011 Feb 16.

Disclosure of Interests: None declared.

DOI: 10.1136/annrheumdis-2021-eular.3130

\section{POS1157 CONCORDANCE BETWEEN THE QUANTIFERON-TB GOLD IN-TUBE AND TUBERCULIN TEST FOR THE DIAGNOSIS OF LATENT TUBERCULOSIS INFECTION IN PATIENTS WITH RHEUMATIC DISEASES}

C. Pávez Perales ${ }^{1}$, A. Quiles Roger², E. Grau García ${ }^{1}$, M. De la Rubia

Navarro $^{1}$, S. Leal Rodriguez ${ }^{1}$, R. Gonzalez Mazario ${ }^{1}$, J. J. Fragío Gil ${ }^{1}$,

C. Alcañiz Escandell ${ }^{1}$, J. Ivorra Cortés ${ }^{1}$, I. Chalmeta Verdejo ${ }^{1}$, L. Gonzalez

Puig $^{1}$, I. Martínez Cordellat ${ }^{1}$, R. Negueroles Albuixech ${ }^{1}$, F. M. Ortiz Sanjuan ${ }^{1}$, J. E. Oller Rodríguez ${ }^{1}$, E. Vicens Bernabeu ${ }^{1}$, C. Nájera Herranz ${ }^{1}$, I. Cánovas Olmos ${ }^{1}$, J. A. Román Ivorra ${ }^{1} .{ }^{1}$ HUP La Fe, Rheumatology Department, Valencia, Spain; ${ }^{2}$ UCV, Medical School, Valencia, Spain

Background: Patients with rheumatic diseases (RD) are at higher risk of latent tuberculosis infection (LTBI) reactivation. To detect and treat it before starting treatment, especially with biological therapies, decrease the reactivation risk. Diagnosis is carried out by the tuberculin skin test (TST) or interferon-gamma release assays (IGRAs), IGRAs might be more specific and sensitive.

Objectives: We aim to analyze the concordance between QuantiFERON-TB Gold In-Tube (QTF) and TST for the diagnosis of LTBI in patients with rheumatic diseases.

Methods: A retrospective observational study was conducted including patients diagnosed with RD screened for LTBI with both TST and QTF (20142018). Demographical and clinical variables at screening and at follow-up were collected. The concordance between both tests has been estimated as categorical variables using Cohen's Kappa test, considering "poor" if it is $\leq 0,20$; "low" if $0,20<k \leq 0,40$, "moderate" if $0,40<k \leq 0,60$, "substantial" if $0,60<k \leq$ 0,80 and "optimal" if $k>0,80$.

Results: 167 patients were included ( $57 \%$ women) with a mean age of $52 \pm 16$ years. $42 \%$ of them had systemic autoimmune diseases, $22 \%$ spondyloarthropathies and $36 \%$ other RD. 2 had history of past active tuberculosis (TB). At the time of screening, $46.11 \%$ were treated with GC.

LTBI was diagnosed in 35 patients: 15 had both QTF and TST positive, 16 only QTF positive and 4 only TST positive. 12 from 31 QTF positive patients were treated with GC at the time of screening. 3 from 19 TST positive patients were treated with $\mathrm{GC}$ at the time of screening.

After LTBI screening 62 patients received biological treatment, 4 of them had both test positive, 6 only QTF positive and 2 only TST positive. 11 received $L T B I$ treatment according to the hospital protocol (isoniazid for 6 to 9 months). 10 completed treatment, 1 did not because of intolerance and did not receive other treatment. 1 patient with only TST positive was considered a false positive and did not receive treatment. During follow-up no TB reactivation was reported.

23 patients with LBTI received treatment other than biological therapy during follow-up, of them 8 received LBTI treatment. There was no TB reactivation during follow up.

The Kappa concordance between QTF and TST was estimated: moderated in the whole sample, poor in the patients treated with GC at screening, and substantial when the patients treated with $\mathrm{GC}$ at screening were excluded. Results are shown in Table 1.
Table 1. Kappa concordance between QTF and TST.

\begin{tabular}{|llll|}
\hline & $\begin{array}{l}\text { TOTAL } \\
\text { SAMPLE }\end{array}$ & $\begin{array}{l}\text { PATIENTS } \\
\text { WITH GC } \\
\text { AT LTBI } \\
\text { SCREENING }\end{array}$ & $\begin{array}{l}\text { PATIENTS } \\
\text { WITHOUT } \\
\text { GC AT LTBI }\end{array}$ \\
SCREENING
\end{tabular}

Conclusion: QTF seems to be the most appropriate LTBI screening test in patients with RD treated with GC. Screening and treatment of LTBI in patients with $R D$ treated with or without biological agents was effective in reducing TB reactivation.

Disclosure of Interests: None declared.

DOI: 10.1136/annrheumdis-2021-eular.3157

\section{\begin{tabular}{|l|l}
\hline POS1158 THE SPECTRUM OF NON-TUBERCULOUS \\
\hline
\end{tabular} OSTEOARTICULAR INFECTIONS IN A RHEUMATOLOGY DEPARTMENT DURING THE YEARS 2010-2020: A RETROSPECTIVE STUDY OF 102 CONSECUTIVE CASES IN A SINGLE CENTER}

M. Chammakhi ${ }^{1}$, C. Bendahmane ${ }^{1}$, F. Kemiche ${ }^{1}$, I. Cerf-Payrastre ${ }^{1}$,

E. Pertuiset ${ }^{1}{ }^{1}$ Centre Hospitalier René Dubos, Rheumatology, Pontoise, France

Background: osteoarticular infections (OAI) are characterized by a large variety of sites, causative microorganisms, source and route of infection, risk factors and also by the fact that several medical specialties are involved in diagnosis and treatment. These characteristics are subject to changes over the time.

Objectives: to describe the spectrum of non-tuberculous OAI in the absence of OA material, including native septic arthritis (SA) and non-postoperative SA or infectious spondylodiscitis (SPDI) during the years 2010-2020. This includes demographics, comorbidities, site of infection, causative microorganisms, source of infection, diagnosis, treatment procedures and mortality.

Methods: medical records of patients aged 18 years old or above who were diagnosed with a non-tuberculous OAI in the Department of rheumatology of our hospital during the 2010-2020 period were selected and retrospectively reviewed. OAl with the following characteristics were excluded: SA on prosthetic joints, postoperative SA or SPDI, osteomyelitis, brucellosis, Lyme disease. Only proven cases where included on the basis of an isolated pathologic organism at the site of infection and/or in the blood (with typical clinical, biological and imaging features).

Results: There were 102 consecutive patients (males $62 \%$ ), aged $62.5 \pm 16.8$ years, with an IMC of $25.1 \pm 5.2$. Peripheral SA where observed in 52 cases, SPDI in 33 cases, non-peripheral SA in 10 cases (sacroiliitis 5, pubic symphysitis 2 , sternoclavicular 2, posterior interapophyseal 1); in 7 patients, there was an association of SPDI and peripheral SA. At least two non-contiguous sites were involved in 22 patients $(21.5 \%)$. The sites of the positive sample where: osteoarticular (synovial fluid, vertebral biopsy) in 47 cases; blood in 28 cases; both of them in 27 cases.

In the 102 cases, 105 microorganisms grew in culture including one Candida glabrata. The following bacteria where responsible in the other cases: 42 Staphylococcus aureus (including 3 cases of methicillin resistant); 7 coagulase-negative Staphylococcus spp.; 6 Group A Streptococcus; 12 group B Streptococcus agalactiae; 8 groups C/G Streptococcus; 3 group D Streptococcus gallolyticus; 2 Streptococcus pneumoniae; 1 Neisseria gonorrhea; 8 enterobacteria; 3 Enterococcus faecalis; 3 Pseudomonas aeruginosa; 3 Haemophilus; 6 others species (2 Parvimonas micra, 1 aerococcus urinae, 1 Nesseiria bacilliformis, 1 bacteroides fragilis, 1 campylobacter fetus).

The most frequent host risk factors were: diabetes (21\%), inflammatory/auto-immune disease (10\%; including 3 rheumatoid arthritis), chronic renal insufficiency 